\title{
COMPARACIÓN ENTRE LA HABILIDAD DE CUIDADO DE CUIDA- DORES DE PACIENTES CON ENFERMEDAD CEREBROVASCULAR Y DIABETES MELLITUS EN SAN JOSÉ DE CÚCUTA, NORTE DE SANTANDER.
}

Por:

Dianne Sofía González E. *, Olga Marina Vega Angarita **

\section{RESUMEN}

Objetivo:

Comparar la habilidad de cuidado de los cuidadores familiares de pacientes por Diabetes Mellitus y Enfermedad Cerebrovascular en San José de Cúcuta, Norte de Santander.

Materiales y Métodos:

Corresponde a un diseño de tipo descriptivo comparativo con abordaje cuantitativo en 128 cuidadores familiares de pacientes (64 por Enfermedad Cerebrovascular y 64 por Diabetes mellitus).

Resultados:

Contienen la descripción de los grupos a estudio en términos de sexo, edad, nivel de escolaridad, estado civil, ocupación, nivel socioeconómico, cuidado desde el momento del diagnóstico, tiempo de cuidado y parentesco con la persona cuidada; nivel de funcionalidad del receptor de cuidado de acuerdo con su capacidad física y cognitiva. El nivel de habilidad total y de sus dimensiones: Conocimiento, Valor y Paciencia se comparan en los dos grupos de cuidadores. El estudio reporto similitud en las características sociodemográficas de la población participante, como en el nivel de funcionalidad de los receptores de cuidado.

\section{Discusión:}

Los hallazgos del estudio confirman lo reportado en la literatura de enfermería respecto a las características sociodemográficas y niveles deficientes en la habilidad de cuidado. En cuanto a los componentes que integran la habilidad de cuidado: conocimiento, valor y paciencia, se encuentran diferencias estadísticamente significativas entre los dos grupos, reportando los cuidadores familiares de pacientes diabéticos mayores niveles de conocimiento y paciencia. Respecto al componente valor no se encontró diferencia significativa entre los grupos a estudio.

PALABRAS CLAVE:

Cuidadores, Habilidad, Enfermedad Cerebrovascular, Diabetes Mellitus

\section{SUMMARY}

Objective :

To compare the care ability among the family caretakers of patients with Mellitus Diabetes and Cerebral Vascular diseases in San Jose de Cucuta, Norte de Santander.

Materials And Methodology:

It corresponds to a descriptive and comparative type of quantitative approach among the family caretakers of 64 Cerebral Vascular disease and 64 Mellitus Diabetes disease patients.

Results:

It contents a description of the study groups about the gender, age, school level, civil status, occupation, socio-economical level, time and care of the patient from diagnosis, kinship, and the level of potential functions on the receptor of the care according to their physical and cognitive capacities. The level of the total ability and its dimensions such as Knowledge, Courage and Patience are compared between the caretakers groups. The study showed similarity in the socio-demographic characteristics of the participants, as of in the level of potential functions on the receptors of the care.

Argument: The findings of the study confirmed what was reported in the nursing literature regarding the socio-demographic characteristics and the shortcomings

*Docente Asociado Facultad Ciencias de la Salud, Universidad Francisco de Paula Santander, Enfermera Magíster Cuidado de enfermería Paciente Crónico, Grupo de Investigación Cuidado de Enfermería. dsgonzal@bari.ufps.edu.co

*** Enfermera Magíster Cuidado de enfermería Paciente Crónico. Docente Asociado Facultad Ciencias de la Salud, Universidad Francisco de Paula Santander, Grupo de Investigación Cuidado de Enfermería.omvega@bari.ufps.edu.co 


\section{COMPARACIÓN ENTRE LA HABILIDAD DE CUIDADO DE CUIDADORES DE PACIENTES CON ENFERMEDAD}

\section{CEREBROVASCULAR Y DIABETES MELLITUS EN SAN JOSÉ DE CÚCUTA, NORTE DE SANTANDER.}

of the ability of the care given. In the components that integrate the ability to take care of the patient such as knowledge, courage and patience, significant statistic differences were found between the two groups, reporting mayor levels of knowledge and patience among the family caretakers of the diabetes patients. Regarding the component of courage, there were no significant differences between the groups of the study.

Key Words:

Caretakers, Ability, Cerebrovascular Accident, Diabetes Mellitus.

\section{INTRODUCCIÓN}

El mundo está experimentando un rápido aumento de los problemas de salud, hasta el punto que las enfermedades crónicas constituyen actualmente más de la mitad de la carga mundial de morbilidad'. Anteriormente las enfermedades infecciosas agudas eran la principal preocupación de los profesionales de la salud en todos los países. Sin embargo, durante el siglo pasado los avances en la medicina, la técnica y la salud pública, en aspectos como vacunaciones, saneamiento, vivienda y educación han contribuido a una disminución de la incidencia de las enfermedades agudas.

Estos avances han supuesto un aumento de la esperanza de vida. Al haber aumentado la longevidad, las personas están expuestas durante más tiempo a los factores de riesgo que favorecen el desarrollo de enfermedades discapacitantes. La enfermedad Cerebrovascular y la Diabetes Mellitus están estrechamen- te ligadas con el envejecimiento.2

La Organización Mundial de la Salud (OMS) ${ }^{3}$ señala que el $60 \%$ de las muertes que ocurren en el mundo se deben en especial la enfermedad Cardiaca y la Cerebrovascular, esta última ocupa un lugar preponderante, ubicándose en el primer lugar de mortalidad durante la década 1985-19954. Lo más relevante son los aspectos cualitativos de la vida en los sobrevivientes; el $90 \%$ de éstos presentan algún tipo de restricción de la capacidad para realizar las actividades de la vida diaria de forma independiente. En numerosos casos la discapacidad se refleja en una situación de dependencia ${ }^{5}$.

"La Diabetes Mellitus actualmente es considerada una pandemia, con mas de 100 millones de casos en todo el mundo, los cuales 13 millones se ubican en América Latina"6. En la población mayor de 60 años, la diabetes y sus complicaciones tardías pueden afectar seriamente el estado de salud funcional. La hipertensión arterial, las neuropatías periféricas, las nefropatías y cardiopatías, hacen que la persona enferma se desenvuelva con dificultad para satisfacer sus necesidades físicas y psicológicas ${ }^{7}$.

La fase de la enfermedad determina la dependencia de la persona cuidada, necesidades de cuidado, y de la habilidad del cuidador. Se categorizaron las enfermedades en cuatro grandes grupos: 1) Aquellas que, para mejorar su pronóstico, requieren una modificación en el estilo de vida de las personas; 2) Las que producen dolor, limitación o mutilación; 3) Las que producen un intenso sentimiento de pérdida o afrontamiento de la muerte y 4) Aquellas que alteran en forma importante la capacidad de socialización del cuidador o del receptor del cuidado 8

\footnotetext{
${ }^{1}$ D WORLD HEALTH REPORT 2004. Changing history. Geneva, World Health organitatión, 2004.

2 ORGANIZACIÓN MUNDIAL DE LA SALUD, El Reto de las enfermedades crónicas: preparación de los profesionales de la salud para el siglo XXI.

Suiza, 2005, p. 17.

3 ORGANIZACIÓN MUNDIAL DE LA SALUD, USA, OPS-OMS, 2004.

${ }^{4}$ RINCON Osorio, Fanny. Propuesta de guía de enfermería del paciente con infarto agudo del miocardio. En: Revista Actualizaciones de enfermería, Bogota. Vol. 7, NN 4, (Diciembre 2004); p.12.

${ }^{5}$ ANZOLA P Elías, GALINSKY David, MORALES M, Fernando, La atención del anciano hoy, Organización Panamericana de la Salud. Publicación Científica № 546 Washington D.C: 109

${ }^{6}$ WHITE, Franklin, NANAN, Debra. Situación de los programas nacionales contra la diabetes en las Américas. OMS, 2000. p. 141

7 BAÑUELOS Barrera Patricia, GALLEGOS Esther C, Autocuidado y control en adultos mayores con Diabetes, En Revista Desarrollo científico de enfermería, México Volumen 9 NN$^{\circ} 4$ (Mayo) 2001, p.101

8 BARRERA O. L, GALVIS L. CR, MORENO F. ME, PINTO A. N, Pinzón R. ML, Romero G. E, Sánchez H. B. La habilidad de cuidado de los cuidadores familiares de personas con enfermedad crónica. Invest. educ. Enferm 2006; 24(1): 36-46
} 


\section{COMPARACIÓN ENTRE LA HABILIDAD DE CUIDADO DE CUIDADORES DE PACIENTES CON ENFERMEDAD CEREBROVASCULAR Y DIABETES MELLITUS EN SAN JOSÉ DE CÚCUTA, NORTE DE SANTANDER.}

La mayor parte de los procesos de enfermedad y de cuidado de personas dependientes se realizan en el ámbito familiar; esto llega a suponer el $88 \%$ de los cuidados que consume el paciente, sólo el 12\% restante de los cuidados es prestado por los servicios de salud, lo cual da idea de la importancia que tienen los cuidadores familiares. ${ }^{8}$

Por cuidador familiar se entiende aquella persona que presta cuidado regular, sin ninguna remuneración ni capacitación formal a otra que por tener una enfermedad o una discapacidad física no puede hacerlo por sí misma9. Ofrecer una atención sin límites, tener un elevado grado de compromiso hacia la tarea caracterizada por el afecto, reciprocidad y obligación son otros aspectos que precisan su definición. ${ }^{10}$

La responsabilidad y tareas de cuidado suponen el desarrollo de habilidades de parte de quien las asume, por lo que el reconocimiento de la misma es importante para promover los apoyos necesarios en su desarrollo. Schumacher, Stewart y Archbold (1998)11 identifican cinco conceptos que representan el hacer bien la entrega de cuidado: maestría en la entrega de cuidado, autoeficacia, competencia, preparación y calidad.

Si bien existe una cantidad significativa de referencias bibliográficas sobre los cuidadores en los países desarrollados, éstas son aún limitadas en países en desarrollo como Colombia. El conocimiento sobre la habilidad de cuidado de los cuidadores familiares en la región es reducido; no se conoce de estudios que hayan revisado dicha temática de acuerdo a la enfermedad que padece el receptor de cuidado, por lo que se consideró de importancia abordar este problema desde la perspectiva de enfermería.
El estudio surge del interés por conocer ¿̇Cómo son las habilidades de cuidado de los cuidadores familiares de personas adultas con Diabetes Mellitus en comparación con las habilidades de cuidado de los cuidadores familiares de personas adultas con Enfermedad Cerebrovascular en San José de Cúcuta, Norte de Santander?

\section{OBJETIVO GENERAL}

Comparar las habilidades de cuidado de los cuidadores familiares de personas adultas por Diabetes con las habilidades de cuidado de los cuidadores familiares de personas adultas por Enfermedad Cerebrovascular del Hospital Universitario Erasmo Meoz y Centro de Rehabilitación del Norte de Santander.

\section{OBJETIVOS ESPECÍFICOS}

- Describir la habilidad de cuidado de los cuidadores familiares de personas adultas por enfermedad por Diabetes Mellitus del Hospital Universitario Erasmo Meoz y Centro de Rehabilitación del Norte de Santander.

- Describir la habilidad de cuidado de los cuidadores familiares de personas adultas por Enfermedad Cerebrovascular del Hospital Universitario Erasmo Meoz y Centro de Rehabilitación del Norte de Santander.

\section{DEFINICIÓN CONCEPTUAL DEL ESTUDIO}

Habilidad de cuidado. Retoma la definición de habilidad de cuidado propuesta por Ngozi O Nkongo (1999). Vista como la manera de relacionarse con el receptor del cuidado basado en los conceptos de cuidado

\footnotetext{
9 SÁNCHEZ HERRERA, Beatriz, Habilidad del cuidado de los cuidadores de personas en situación crónica de enfermedad. El arte y la ciencia del Cuidado. Facultad de Enfermería, Universidad Nacional de Colombia. Unibiblos, 2002, p. 273

10 MARCO Navarro Esther. Calidad de Vida del paciente Hemipléjico Vascular, Departamento de Medicina, Universitat Autonoma de Barcelona, [Serial online] Septiembre 2004, Disponible en URL: http://www.tdx.cbuc.es/TESIS_UAB/AVAILABLE/TDX-0728105133440//emn l de 1.pdf.

11 SCHUMACHER K, STEWART B y ARCHBOLD P, Conceptualización y medición de la ejecución de ser un buen cuidador familiar. En: Journal of Nursing Scholarship. New York.Vol. 30, N 1 (1998) p. 6
} 


\section{COMPARACIÓN ENTRE LA HABILIDAD DE CUIDADO DE CUIDADORES DE PACIENTES CON ENFERMEDAD}

\section{CEREBROVASCULAR Y DIABETES MELLITUS EN SAN JOSÉ DE CÚCUTA, NORTE DE SANTANDER.}

como la relación que debe poseer conocimiento, cambios de ritmo, paciencia, honestidad, confianza, humildad, esperanza y coraje; y habilidad como lo que experimenta el cuidador, una sensación de pertenencia y vinculo estable, determinado en el grado de desarrollo de acuerdo con tres elementos: conocimiento, valor y paciencia.

\section{Cuidador Familiar.}

Entendido como la persona que presta cuidado regular, sin ninguna remuneración ni capacitación formal, a otra que por tener una enfermedad o una discapacidad física, tiene dificultades para asumir su propio cuidado especialmente en lo relacionado con actividades de la vida diaria y actividades básicas cotidianas.

Persona en situación de enfermedad Cerebrovascular.

Identifica a la persona a quien su cuidador reconoce como alguien que padece limitación física, sensitiva y de comunicación de tal magnitud que le genera dependencia y que requiere de su cuidado.

Persona con Diabetes Mellitus.

Persona con enfermedad metabólica que ha desarrollado complicaciones agudas y crónicas, de carácter discapacitante cuya aparición y progresión afectan significativamente su calidad de vida y capacidad productiva.

\section{MATERIALES Y MÉTODOS}

El estudio utilizó un diseño de tipo descriptivo comparativo con abordaje cuantitativo. Los sujetos a estudio lo conformaron 128 cuidadores familiares de pacientes; específicamente 64 por Enfermedad Cerebrovascular y 64 por Diabetes Mellitus. La muestra se captó por conveniencia al constatar el cumplimiento de criterios de inclusión: Ser adulto y cuidador de una persona con Diabetes Mellitus y Enfermedad Cerebrovascular por más de tres meses. El Hospital Universitario Erasmo Meoz y Centro de
Rehabilitación se constituyeron en escenarios del estudio efectuado durante los meses Diciembre del 2005 a Junio de 2006.

La información se recolectó por medio de tres instrumentos: Caracterización de los cuidadores, Perfil PULSES y el Inventario para la Habilidad de Cuidado. En la descripción de las características sociodemográficas de los cuidadores participantes se utilizó un cuestionario diseñado por la Facultad de Enfermería de la Universidad Nacional de Colombia que identifica el sexo, edad del cuidador y del paciente, nivel de escolaridad, estado civil, ocupación, nivel socioeconómico, cuidado desde el momento del diagnóstico, tiempo de cuidado y parentesco con la persona cuidada.

La determinación del nivel de funcionalidad de los receptores de cuidado en términos de su capacidad física y cognitiva fue medida a través del perfil "PULSES" creada por Moskowitz en 1957. Esta escala refleja los niveles de funcionalidad en seis parámetros: P: Estabilidad de la patología o condición física, U: Utilización de miembros superiores, L: Locomoción - utilización de miembros inferiores, S: Función sensorial, E: Eliminación o control de esfínteres, S: Capacidad de socializar; con una graduación tipo likert; donde uno (1) equivale a independiente y cuatro (4) a totalmente dependiente.

Finalmente en la evaluación del grado de habilidad de cuidado, se utilizó el instrumento diseñado por la Ngozi O. Nkongho "Inventario para la habilidad de cuidado", denominado (CAl) por sus iniciales en ingles Caring Hability Inventory.

Su ponderación permitió valorar el nivel de habilidad total y de sus dimensiones según Conocimiento, Valor y Paciencia. Estructuralmente dicho instrumento con un grado de validez de 0.80 y confiabilidad de 0.85 se compone de 37 preguntas, con escala de respuesta tipo Likert que van de 1 a 7 , en donde 1 equivale a "totalmente en desacuerdo" y 7 "totalmente de acuerdo". 


\section{COMPARACIÓN ENTRE LA HABILIDAD DE CUIDADO DE CUIDADORES DE PACIENTES CON ENFERMEDAD}

CEREBROVASCULAR Y DIABETES MELLITUS EN SAN JOSÉ DE CÚCUTA, NORTE DE SANTANDER.

\section{MÉTODOS ESTADÍSTICOS}

La información recolectada se incorporó a la matriz Excel previamente diseñada. Con el fin de identificar diferencias significativas en cuanto a lo niveles medianos de las habilidades de cuidado general y por cada componente entre los grupos de cuidadores a estudio, de forma computarizada se realizo la prueba de Mann Whitney con un nivel de significancia de 0.05 .

\section{ASPECTOS ÉTICOS}

Desde lo ético se respeto la libre participación de los cuidadores, se contó con la aprobación de los respectivos Comités Técnico Científico de las instituciones sedes, la información obtenida en los instrumentos se documentó de manera anónima y con fines únicos para el estudio.

\section{RESULTADOS}

\section{Características sociodemográficas}

Al analizar los datos de los dos grupos a estudio se encuentra que al igual que en estudios realizados en otros contextos y lo reportado en la literatura de enfermería, el rol de cuidador familiar es desempeñado principalmente por mujeres (84.4\%, 76.6\%): esposas, hijas, hermanas y nueras, ubicadas en su mayoría en el rango de edad de 36 a 59 años (53.12\% y $53.90 \%)$, se destaca un porcentaje de cuidadores mayores de 60 años $(21.87 \%, 23.43 \%)$.

Respecto a la edad del paciente en ambos casos el mayor porcentaje $(56.25 \%, 62.5 \%)$ corresponde al rango de edad mayor que el cuidador (Tabla 1 ).

En relación al tiempo en el ejercicio del rol de los cuidadores con Enfermedad Cerebrovascular, se encontró que el 34.37\% llevan de 3 a 6 meses, seguido de un $31.25 \%$ con mas de 37 meses. Por el contrario, en el grupo de pacientes diabéticos un porcentaje re- presentativo de cuidadores (60.93\%) lleva mas de 37 meses en el desempeño del rol, y el $4.68 \%$ de 3 a 6 meses.

Respecto a la característica único cuidador, los resultados para los dos grupos muestran un mayor porcentaje $(51.6 \%, 64.1 \%)$ de cuidadores que cuentan con redes de apoyo familiar que comparten la responsabilidad de atención del enfermo.

En el estudio se resalta que el $48.4 \%$ y el $35.9 \%$ de la población manifiesta el desempeño del rol de manera solitaria.

Sobre el nivel de funcionalidad de los receptores de cuidado se encontró que un porcentaje significativo $(90.6 \%$, y $93.8 \%)$ presentan un compromiso de la funcionalidad entre moderado y severo (Tabla 1 )..

\section{HABILIDAD DE CUIDADO DE LOS CUIDADORES}

En relación con los cuidadores de enfermos neurovasculares, según el nivel de habilidad de cuidado general, la calificación promedio encontrada fue de 182 puntos con una desviación estándar de 9.6 puntos. El nivel de habilidad total fue óptimo en el $28.1 \%$ de los cuidadores de este grupo, medio en el $42.2 \%$ de los casos y bajo en el $29.7 \%$. En cuanto a las subescalas, se encontró un nivel alto de conocimiento en el $26.6 \%$ de los cuidadores, medio en el $51.6 \%$ y bajo en el $21.9 \%$ de ellos. En la subescala valor, el $28.1 \%$ tiene un nivel alto, un $37.5 \%$ un nivel medio y el $34.4 \%$ bajo. En la paciencia se encontró un nivel alto en el $28.1 \%$, medio en el $46.9 \%$ y bajo en el $25.0 \%$ de los cuidadores (Tabla 2).

Respecto al grupo de cuidadores familiares de enfermos por Diabetes Mellitus, la calificación promedio de habilidad de cuidado general (CAl Total) fue de 186 con una desviación estándar de 11.7. En 


\section{COMPARACIÓN ENTRE LA HABILIDAD DE CUIDADO DE CUIDADORES DE PACIENTES CON ENFERMEDAD}

\section{CEREBROVASCULAR Y DIABETES MELLITUS EN SAN JOSÉ DE CÚCUTA, NORTE DE SANTANDER.}

cuanto al nivel de habilidad de cuidado total, se encontró alto en el $29.7 \%$, medio para el $37.5 \%$ y bajo en el $32.8 \%$ de ellos. El conocimiento presento un nivel alto en el $26.6 \%$ de los cuidadores, medio en el $54.7 \%$ y bajo en el $18.8 \%$. El nivel de valor fue alto en el $31.3 \%$ de los casos, y en las categorías media y baja el $34.4 \%$ respectivamente. La paciencia fue alta en el $46.9 \%$, media en el $32.8 \%$ y baja en el $20.3 \%$ de ellos. (Tabla 2 )

Al comparar el nivel de habilidad total y por categorías de los grupos a estudio, se encontraron dife- rencias estadísticamente significativas en cuanto a los niveles medianos de conocimiento y paciencia entre ambos grupos, observándose que el nivel mediano de conocimiento y paciencia de cuidadores de pacientes Diabéticos es mayor que la de cuidadores de familiares con Enfermedad Cerebrovascular $(Z=-2,16$, $-3,70)$. No se encontraron diferencias estadísticamente significativas en cuanto al nivel mediano del componente valor entre ambos grupos de cuidadores a estudio $(Z=1,28)$. (Tabla 3).

Tabla 1. DISTRIBUCIÓN PORCENTUAL DE LAS CARACTERÍSTICAS DE LOS CUIDADORES FAMILIARES Y RECEPTORES DE CUIDADO

\begin{tabular}{|c|c|c|c|c|c|}
\hline \multirow{2}{*}{\multicolumn{2}{|c|}{ CARACTERISTICA }} & \multicolumn{2}{|c|}{ ENFERMEDAD CEREBROVASCULAR } & \multirow{2}{*}{$\begin{array}{c}\text { DIABETES } \\
\mathbf{N}^{\circ}\end{array}$} & \multirow{2}{*}{$\begin{array}{c}\text { MELLITUS } \\
\%\end{array}$} \\
\hline & & $\mathbf{N}^{\circ}$ & $\%$ & & \\
\hline \multirow[t]{2}{*}{ SEXO } & Hombres & 10 & 15.6 & 15 & 23.43 \\
\hline & Mujeres & 54 & 84.4 & 49 & 76.6 \\
\hline \multirow{3}{*}{ EDAD } & 18-35 años & 16 & 25.0 & 13 & 22.65 \\
\hline & 36-59 años & 34 & 53.12 & 35 & 53.90 \\
\hline & 60 y mas años & 14 & 21.87 & 16 & 23.43 \\
\hline \multirow{3}{*}{ EDAD DEL PACIENTE } & $>$ que el cuidador & 36 & 56.25 & 40 & 62.5 \\
\hline & $=$ al cuidador & 8 & 12.5 & 13 & 20.31 \\
\hline & $<$ que el cuidador & 20 & 31.25 & 11 & 17.18 \\
\hline \multirow{6}{*}{ PARENTESCO } & Esposa(o) & 16 & 25.0 & 11 & 17.2 \\
\hline & Madre/padre & 5 & 7.8 & 2 & 3.1 \\
\hline & Hija & 25 & 39.1 & 27 & 42.2 \\
\hline & Abuela & 3 & 4.7 & 2 & 3.1 \\
\hline & Amiga(o) & 1 & 1.6 & 4 & 6.25 \\
\hline & \begin{tabular}{|l|} 
Otro \\
\end{tabular} & 14 & 21.9 & 18 & 28.1 \\
\hline \multirow{4}{*}{$\begin{array}{l}\text { TIEMPO EN EL EJER- } \\
\text { CICIO DEL CUIDADO }\end{array}$} & $3-6$ meses & 22 & 34.37 & 3 & 4.68 \\
\hline & 7- 18 meses & 12 & 18.75 & 9 & 14.06 \\
\hline & 19 a 36 meses & 10 & 15.62 & 13 & 20.31 \\
\hline & 37 y más & 20 & 31.25 & 39 & 60.93 \\
\hline \multirow[t]{2}{*}{ UNICO CUIDADOR } & $\mathrm{SI}$ & 31 & 48.4 & 23 & 35.9 \\
\hline & $\mathrm{NO}$ & 33 & 51.6 & 41 & 64.1 \\
\hline \multirow{3}{*}{ PULSES } & Compromiso Leve & 6 & 9.38 & 4 & 6.3 \\
\hline & Compromiso Moderado & 10 & 15.6 & 20 & 31.3 \\
\hline & $\begin{array}{l}\text { Compromiso } \\
\text { Severo }\end{array}$ & 48 & 75.0 & 40 & 62.5 \\
\hline
\end{tabular}

Fuente: Datos de la investigación 


\section{COMPARACIÓN ENTRE LA HABILIDAD DE CUIDADO DE CUIDADORES DE PACIENTES CON ENFERMEDAD} CEREBROVASCULAR Y DIABETES MELLITUS EN SAN JOSÉ DE CÚCUTA, NORTE DE SANTANDER.

Tabla 2. DISTRIBUCIÓN PORCENTUAL DEL NIVEL DE HABILIDAD DE CUIDADO GENERAL Y POR COMPONENTES.

\begin{tabular}{|c|c|c|c|c|c|}
\hline \multirow{2}{*}{\multicolumn{2}{|c|}{ NIVEL DE HABILIDAD POR CATEGORIA }} & \multicolumn{2}{|c|}{ ENFERMEDAD CEREBROVASCULAR } & \multirow{2}{*}{$\begin{array}{l}\text { DIABETES } \\
\mathbf{N}^{\circ}\end{array}$} & \multirow{2}{*}{$\begin{array}{c}\text { MELLITUS } \\
\quad \%\end{array}$} \\
\hline & & $\mathbf{N}^{\circ}$ & $\%$ & & \\
\hline \multirow{3}{*}{$\begin{array}{l}\text { HABILIDAD DE } \\
\text { CUIDADO GENERAL. } \\
\text { ( CAI TOTAL) }\end{array}$} & BAJA & 19 & $29.7 \%$ & 21 & $32.8 \%$ \\
\hline & MEDIA & 27 & $42.2 \%$ & 24 & $37.5 \%$ \\
\hline & ALTA & 18 & $28.1 \%$ & 19 & $29.7 \%$ \\
\hline \multirow[t]{3}{*}{ CONOCIMIENTO } & BAJA & 14 & $21.9 \%$ & 12 & $18.8 \%$ \\
\hline & MEDIA & 33 & $51.6 \%$ & 35 & $54.7 \%$ \\
\hline & ALTA & 17 & $26.6 \%$ & 17 & $26.6 \%$ \\
\hline \multirow[t]{3}{*}{ VALOR } & BAJA & 22 & $34.4 \%$ & 22 & $34.4 \%$ \\
\hline & MEDIA & 24 & $37.5 \%$ & 22 & $34.4 \%$ \\
\hline & ALTA & 18 & $28.1 \%$ & 20 & $31.33 \%$ \\
\hline \multirow[t]{3}{*}{ PACIENCIA } & BAJA & 16 & $25.0 \%$ & 14 & $20.3 \%$ \\
\hline & MEDIA & 30 & $46.9 \%$ & 36 & $32.8 \%$ \\
\hline & ALTA & 18 & $28.1 \%$ & 14 & $46.9 \%$ \\
\hline
\end{tabular}

Fuente: Datos de la investigación

Tabla 3. RESULTADOS DE LA PRUEBA MANN-WHITNEY POR CADA COMPONENTE DE HABILIDAD DE CUIDADO

\begin{tabular}{|l|l|l|l|l|}
\multicolumn{1}{c|}{ COMPONENTE } & \multicolumn{1}{c|}{ VALOR PARA S } & \multicolumn{1}{c|}{ VALOR PARA T } & \multicolumn{1}{c|}{ VALORES CRITICOS } \\
\hline Conocimiento & 3673,5 & 1593,5 & $-2,17$ & $(-1,96>=Z>=1,96)$ \\
\hline Paciencia & 3351,5 & 1271,5 & $-3,70$ & $(-1,96>=Z>=1,96)$ \\
\hline Valor & 4397,5 & 2317,5 & 1,28 & $(-1,96>=Z>=1,96)$ \\
\hline Total & 3807,5 & 1727,5 & $-1,53$ & $(-1,96>=Z>=1,96)$ \\
\hline
\end{tabular}

Fuente. IDEM. Tabla 2

\section{DISCUSIÓN Y ANÁLISIS}

En los grupos a estudio se encontró que el cuidado es desempeñado principalmente por mujeres: esposas, hijas, hermanas y nueras, resultado que coincide con numerosos trabajos previos en el contexto latinoamericano y nacional en el que ratifican que la responsabilidad del cuidado de un familiar enfermo recae principalmente en las personas de genero femenino. En el contexto regional, estudios efectuados en el 200512131415 sobre la habilidad del cuidado coinciden en este aspecto. La mujer se constituye en el modelo más típico de cuidadora en el contexto Nortesantandereano.

\footnotetext{
12 VEGA Angarita, Olga Marina, Habilidad del cuidado de los cuidadores primarios de adultos discapacitados por enfermedad Cerebrovascular del Norte de Santander 2005, Trabajo de grado para obtener el titulo de Magíster en enfermería con énfasis al paciente Crónico, Universidad Nacional de Colombia-Universidad Francisco de Paula Santander ${ }^{2}$ ORGANIZACION MUNDIAL DE LA SALUD, El Reto de las enfermedades crónicas: preparación de los profesionales de la salud para el siglo XXI. Suiza, 2005, p. 17

13 GONZALEZ, Escobar Dianne Sofía, Habilidad del Cuidado de cuidadores familiares de personas en situación crónica de enfermedad por diabetes Mellitus 2005, Tra bajo de grado para obtener el titulo de Magíster en enfermería con énfasis al paciente Crónico, Universidad Nacional de Colombia-Universidad Francisco de Paula Santander

14 RAMIREZ Ordóñez Ma Mercedes, Habilidad de cuidado de los cuidadores principales en el paciente con cáncer gastrointestinal 2005 , Trabajo de grado para obtener el titulo de Magíster en enfermería con énfasis al paciente Crónico, Universidad Nacional de Colombia-Universidad Francisco de Paula Santander.
} 


\section{COMPARACIÓN ENTRE LA HABILIDAD DE CUIDADO DE CUIDADORES DE PACIENTES CON ENFERMEDAD}

\section{CEREBROVASCULAR Y DIABETES MELLITUS EN SAN JOSÉ DE CÚCUTA, NORTE DE SANTANDER.}

Se identifica en los resultados una participación representativa de hombres como cuidadores, lo cual puede estar relacionado con las transformaciones sociales y roles que está viviendo nuestra sociedad, derivadas de una mayor participación de la mujer en el ámbito laboral16, altas tasas de desempleo, movilización y cambio del entorno natural de la familia y en su estructura, vinculación del padre en los procesos de crianza y otras tareas calificadas como "domésticas" y "femeninas".

Los cuidadores se encuentran en su mayoría en el rango de edad de 36 a 59 años, etapa en la que los cuidadores se encuentran en su periodo de mayor productividad y desarrollo. La situación de enfermedad los expone a la dificultad de compatibilizar el cuidado con otras actividades. El problema de la simultaneidad de las obligaciones y responsabilidades de cuidado es un factor de desestabilización emocional y aumento de tensiones y preocupación en el cuidador ${ }^{17}$.

Es igualmente significativo para ambos grupos, el porcentaje de cuidadores mayores de 60 años que tienen la responsabilidad de cuidado de una persona con alto grado de dependencia. Situación que se hace critica en el caso de las mujeres que envejecen, muchas estarán atendiendo a sus esposos y ayudando a hijos y nietos, contando con menos apoyo familiar que les ayude a ocuparse de sus padres enfermos ${ }^{18}$.

Otro aspecto relevante sobre la edad es que los receptores de cuidado son mayores que su cuidador. Este dato coincide con lo obtenido en un estudio comparativo en tres países ${ }^{19}$, que reporto un comportamiento relativamente homogéneo al encontrado. Dada la estrecha relación de estas enfermedades con el envejecimiento, puede concluirse que el número de cuidadores jóvenes con parentescos de hijas seguirá aumentando en los próximos años.

En relación con el tiempo de ejercicio del rol se destaca la corta experiencia de 3 a 6 meses de los cuidadores de pacientes con Enfermedad Cerebrovascular, hallazgo congruente con la presentación abrupta del cuadro clínico y de las secuelas residuales que ocasionan entre otras la perdida de la autonomía física del paciente y demanda la asistencia continua de su cuidador. Lo encontrado en el estudio es referido por la literatura que muestra que la cantidad y tipo de ayuda varía en función del grado y de la causa que haya determinado dependencia de la persona cuidada. Cuanto mayor sea el grado de dependencia, mayor será también la cantidad de tiempo y de esfuerzo que tendrá que dedicar el cuidador "inexperto" a la tarea de cuidar a su familiar. Por el contrario, en el grupo de pacientes diabéticos un porcentaje representativo de cuidadores lleva más de 37 meses en el desempeño del rol. Las complicaciones tardías de tipo ocular (retinopatía, cataratas), cardiovascular (arteriopatia coronaria), neuropatía y nefropatia hacen que el enfermo se desenvuelva con dificultad para satisfacer sus necesidades físicas y psicológicas, lo que representa para los cuidadores un aumento progresivo de la demanda de cuidado.

En los receptores de cuidado se reporta el compromiso entre moderado y severo en su funcionalidad, lo cual es consistente con la literatura consultada

\footnotetext{
${ }^{15}$ CHÁVEZ Cañas, Wilmer; ESTÉVEZ López, Erica P.; RUEDA Barranco, Julieth J. "Grado de preparación para el cuidado en casa de cuidadores familiares de adultos en situación de enfermedad crónica discapacitante neurovascular de la ESE Hospital Universitario Erasmo Meoz durante el período comprendido entre marzo y julio de 2005". Trabajo de grado para optar al título de enfermera(o), Programa de Enfermería, Facultad de Ciencias de la Salud, Universidad Francisco de Paula Santander, p.95

${ }^{16}$ CELMA Vicente Matilde, Cuidados Informales en el Medio Hospitalario, En: Revista Rol de enfermería, Volumen 24 (7-8) JulioAgosto 2001, España, P. 504

17 QUERO Rufián Aurora, Los cuidadores en el Hospital Ruiz de Alda en Granada, [Tesis Doctoral], Universidad de Granada España, http://hera.ugr.es/tesisugr/16683134.pdf. p. 52

18 ANZOLA P Elías, GALINSKY David, MORALES M, Fernando, Op Cite p.366

19 BARRERA Ortiz, Lucy, BLANCO De Camargo Lidia, FIGUEROA Ingrid P, et al. Habilidad de Cuidadores familiares con enfermedad crónica. Mirada Internacional. En revista Aquichan. 2006, vol.6, no. 1 (6), p 22-33
} 


\section{COMPARACIÓN ENTRE LA HABILIDAD DE CUIDADO DE CUIDADORES DE PACIENTES CON ENFERMEDAD CEREBROVASCULAR Y DIABETES MELLITUS EN SAN JOSÉ DE CÚCUTA, NORTE DE SANTANDER.}

y se asocia a las características propias de la patología que padecen y la etapa de la misma en el momento de realización del estudio. Estas características representan claramente las implicaciones para el cuidador en cuanto a demanda prolongada de cuidado, número de tareas, tiempo y recursos para mantener o alcanzar el mejor nivel bienestar posible para la persona cuidada.

De acuerdo a los resultados en la habilidad de cuidado total en los dos grupos, es evidente que la mayoría de los cuidadores responsables de los familiares enfermos no reúnen las condiciones que se requieren para la prestación del cuidado. Aspecto que los hace más vulnerables a experimentar los efectos negativos de la experiencia de cuidado que afectan no solo su salud en diferentes dimensiones sino la relación misma de cuidado y el bienestar de la persona enferma. Hallazgo que coincide con otros estudios nacionales e internacionales que reportan que en la mayor parte de los casos los cuidadores de enfermos asumen esta tarea sin las competencias requeridas. 202122 La etapa del ciclo vital de los cuidadores, el nivel de funcionalidad del enfermo, la diversidad en el tiempo de ejercicio del cuidado pueden ser factores intervinientes que expliquen el nivel de desarrollo de habilidad alcanzado.

En cuanto a los componentes que integran la habilidad de cuidado se encontró diferencias estadísticamente significativas entre los dos grupos, reportando los cuidadores de pacientes diabéticos mayores niveles de conocimiento y paciencia. En cuanto al componente valor no se encontró diferencia significativa entre los grupos a estudio.

En el grupo de cuidadores de pacientes con enfermedad Cerebrovascular se encuentra como aspecto relevante en el análisis de los ítems afectados que valoran el componente conocimiento, la expresión de la limitación de tiempo, problema en la aceptación del otro y expresión de sentimientos. Esto puede representar de acuerdo a los planteamientos de N. Nkongo(1.999)23, dificultades en el proceso de reconocimiento y comprensión de la persona cuidada como algo especial, con necesidades únicas derivadas de la situación de salud en la que se encuentra.

Igualmente refleja el enfrentamiento de los cuidadores a sentimientos de impotencia generados por la responsabilidad de cuidar prolongadamente a un familiar discapacitado. Una de las características que definen la enfermedad es su forma súbita de aparición. La consecuencia más dramática es el cambio radical del proyecto de vida emprendido por la persona afectada y su cuidador, este último atraviesa por diferentes etapas que van desde la incertidumbre inicial y el desconocimiento del pronostico, hasta el afrontamiento de los cambios propios a la convivencia con una persona con discapacidad. ${ }^{24}$

Por el contrario, los cuidadores de diabéticos manifiestan la capacidad de aceptación de las otras personas tal como son, de expresar sus sentimientos libremente en la relación de cuidado y reconocimiento de que las personas requieren de privacidad

20 BARRERA O, Lucy, PINTO A, Natividad, SANCHEZ H, Beatriz. Habilidad de Cuidado de Cuidadores Familiares de personas con enfermedad Crónica: Comparación de Géneros. En Revista: Actualizaciones de Enfermería 2006,9(2):9 -13. Fundación Santa Fe de Bogotá. Disponible en URL: http://encolombia.com/medicina/enfermeria/Enfermeria9206-Habilidad.htm

21 BARRERA Ortiz, Lucy, BLANCO De Camargo Lidia, FIGUEROA Ingrid P, et al. Op. Cite p. 28-29

22 GALVIS LOPEZ, Clara Rocio, PINZON ROCHA, Maria Luisa, ROMERO GONZALEZ, Esperanza. Comparación entre la Habilidad de Cuidado de Cuidadores de personas en situación de enfermedad crónica en Villavicencio. En: Revista Avances en Enfermería Volumen XXII No 1 (Enero a Junio) 2004. P. 16

${ }^{23} \mathrm{NKONGHO}, \mathrm{NGOZI}$ O. Inventario para la habilidad de cuidado. En Strickland O. Waltz C. Measurement of Nursing Outcomes. Traducción Facultad de Enfermería. Universidad Nacional de Colombia. 1.999. P. 5

${ }^{24}$ QUEMADA José Ignacio, Modelo de Atención a las personas con daño cerebral, Serie colección de documentos Técnicos, Instituto de Mayores de Servicios Sociales, [Serial Online] Primera Edición [ citado Junio2007], España, Disponible en URL: http://www.imsersomayores.csic.es/documentos/ documentos/mtas-atencion-01.pdf, p. 64 


\section{COMPARACIÓN ENTRE LA HABILIDAD DE CUIDADO DE CUIDADORES DE PACIENTES CON ENFERMEDAD}

\section{CEREBROVASCULAR Y DIABETES MELLITUS EN SAN JOSÉ DE CÚCUTA, NORTE DE SANTANDER.}

para pensar y sentir. Según Fitzgerald $(2000)^{25}$, el conocimiento se considera un proveedor de estructura que disminuye en los cuidadores la incertidumbre y permite el desarrollo de un mapa cognitivo para la continua interpretación de los eventos y toma de decisiones en la acción de cuidado.

Con relación al nivel de paciencia de cuidadores de pacientes con enfermedad Cerebrovascular se identifica que solo un bajo porcentaje cuenta con la paciencia requerida para adaptarse y afrontar el rol de cuidador que viene desempeñando. Del análisis de los ítems que valoran esta subescala, se identifica en algunos cuidadores disminución de la tolerancia, autoexploración y reconocimiento de la necesidad de tiempo para el cambio y el aprendizaje que demanda la experiencia de cuidado; de igual forma, manifiestan como muchas de las situaciones que enfrentan en el desempeño de su rol, no están sujetas a su elección.

En el grupo de cuidadores de diabéticos se encontró una proporción considerable en la categoría alta, es decir, estos cuidadores han alcanzado la Paciencia que les permite desarrollar un mayor nivel de tolerancia a los cambios y a la desorganización que el rol de cuidador genera en su cotidianidad, proyectos y desarrollo personal. Los cuidadores de este grupo expresan el reconocimiento de la necesidad de cambio, admiración por las personas calmadas y pacientes, el respeto por los sentimientos y actitudes de los otros, así como la disponibilidad para asumir el compromiso en el desempeño del rol.

\section{CONCLUSIONES}

En cuanto a las características sociodemográficas de los cuidadores familiares participantes, el estudio demostró que los cuidadores son principalmente mujeres, resultado que confirma lo reportado en la literatura nacional e internacional que documentan el cuidado como una función de genero muy arraigada en las diferentes culturas. De igual forma, se observa que el mayor número de cuidadores pertenecen a la generación intermedia, por encontrarse en edades entre los 36 y 59 años, lo que les representa simultáneamente grandes fortalezas y riesgos en el desempeño del rol de cuidador, por lo que deben ser apoyados para mejorar sus habilidades y capacidad de afrontamiento.

Se observa en el estudio que los cuidadores familiares son principalmente hijas y esposas(o) del receptor de cuidado, seguido de otro tipo de filiación familiar, principalmente hermanas(os) y nueras. El tipo de relación existente entre las personas explica características anteriormente descritas como las del tiempo en el desempeño del rol, cuidado desde el diagnóstico de la enfermedad y horas al día dedicadas al cuidado. Igualmente el parentesco ya sea de sangre, por matrimonio o por adopción son factor importante en la relación de cuidado; de acuerdo a él existen diferencias en la forma en que se vive y acepta la situación de cuidado.

Como característica de los receptores de cuidado, estos son principalmente adultos mayores que presentan en su funcionalidad un compromiso entre moderado y severo, lo cual es consistente con la literatura y se asocia a las características propias de la patología que padecen y la etapa de la misma en el momento de la realización del estudio. Estas características representan claramente las implicaciones para el cuidador en cuanto a demanda de cuidado, mayor numero de tareas, tiempo y recursos para mantener - alcanzar el mejor nivel bienestar posible para la persona cuidada y lo expone a una mayor carga en el cuidado.

Al describir y comparar la Habilidad de Cuidado de los cuidadores familiares de personas con

${ }^{25}$ FITZGERALD MILLER, Judith. Recursos de Poder del Cliente. Philadelphia. Davis, 2000. P.3-16 


\section{COMPARACIÓN ENTRE LA HABILIDAD DE CUIDADO DE CUIDADORES DE PACIENTES CON ENFERMEDAD} CEREBROVASCULAR Y DIABETES MELLITUS EN SAN JOSÉ DE CÚCUTA, NORTE DE SANTANDER.

enfermedad Cerebrovascular y Diabetes Mellitus se observa un comportamiento similar en cuanto a habilidad total y su distribución en el componente valor. Se encontraron diferencias estadísticamente significativas entre los dos grupos, reportando en los cuidadores familiares de pacientes diabéticos mayores niveles de conocimiento y paciencia. Lo encontrado en el cotidiano del cuidador de diabéticos representa la continua interpretación de los eventos, de la toma de decisiones en la acción de cuidado, y del mayor grado de tolerancia a los cambios que el rol genera en su vida, proyectos y desarrollo personal.

Sin embargo, en los cuidadores de familiares con enfermedad Cerebrovascular implica la dedicación considerable de tiempo y energía en la atención de complejas actividades no siempre cómodas y agradables, ni previstas a su libre elección ${ }^{26}$, una experiencia ingrata y solitaria que termina por reducir los niveles de paciencia y del conocimiento de sí mismo.

La información recolectada hasta el momento alrededor de las características, condiciones y habilidad de cuidado de los cuidadores familiares hace evidente la necesidad de direccionar el trabajo de enfermería al cuidado de este grupo a través de la implementación de programas de consejería, apoyo, educación y asistencia, como estrategia de preparación y atención en el rol de cuidador a desempeñar.

En la región se hace necesario la creación de programas y servicios de apoyo a los cuidadores familiares, especialmente en Instituciones que como el Hospital Universitario Erasmo Meoz, prestan servicios principalmente a población del régimen subsidiado y vinculada del departamento.

\section{AGRADECIMIENTOS}

A la Vicerrectoria Asistente de Investigación y Extensión de la Universidad Francisco de Paula Santander por la cofinanciación del Proyecto No 030, al Hospital Universitario Erasmo Meoz, y Centro de Rehabilitación Cardioneuromuscular escenarios del estudio, a los estudiantes de VIII semestre del Programa de Enfermería y a los cuidadores participantes por su valiosa colaboración en la comprensión de una problemática por ellos enfrentada y demanda de la atención oportuna.

\section{REFERENCIAS BIBLIOGRÁFICAS}

1. WORLD HEALTH REPORT 2004. Changing history. Geneva, World Health Organitatión, 2004.

2. ORGANIZACIÓN MUNDIAL DE LA SALUD, El Reto de las enfermedades crónicas: preparación de los profesionales de la salud para el siglo XXI. Suiza, 2005, p. 17.

3. ORGANIZACIÓN MUNDIAL DE LA SALUD, USA, OPS-OMS, 2004.

4. RINCON Osorio, Fanny. Propuesta de guía de enfermería del paciente con infarto agudo del miocardio. En: Revista Actualizaciones de enfermería, Bogota. Vol. 7, No 4, (Diciembre 2004); p. 12.

5. ANZOLA P Elías, GALINSKY David, MORALES M, Fernando, La atención del anciano hoy, Organización Panamericana de la Salud. Publicación Científica No 546 Washington D.C: 109

6. WHITE, Franklin y NANAN, Debra. Situación de los programas nacionales contra la Diabetes en las Américas. OMS, 2000. p. 141.

${ }^{26}$ MARCO Navarro Esther, Calidad de vida del cuidador del hemipléjico vascular, Facultad de Medicina Universitat Autonoma de Barcelona, España 2004, Op. Cite. P. 61 


\section{COMPARACIÓN ENTRE LA HABILIDAD DE CUIDADO DE CUIDADORES DE PACIENTES CON ENFERMEDAD}

\section{CEREBROVASCULAR Y DIABETES MELLITUS EN SAN JOSÉ DE CÚCUTA, NORTE DE SANTANDER.}

7. BAÑUELOS Barrera Patricia, GALLEGOS Esther C, Autocuidado y control en adultos mayores con Diabetes, En Revista Desarrollo científico de enfermería, México Volumen 9 № 4 (Mayo) 2001, p. 101

8. BARRERA O. L, GALVIS L. CR, MORENO F. ME, Pinto A. N, PINZÓN R. ML, Romero G. E, SÁNCHEZ H. B. La habilidad de cuidado de los cuidadores familiares de personas con enfermedad crónica. En Invest. Educ. Enferm 2006; 24(1): 36-46

9. SÁNCHEZ HERRERA, Beatriz, Habilidad del cuidado de los cuidadores de personas en situación crónica de enfermedad. El arte y la ciencia del Cuidado. Facultad de Enfermería, Universidad Nacional de Colombia. Unibiblos, 2002, p. 273

10. MARCO Navarro Esther. Calidad de Vida del paciente Hemipléjico Vascular, Departamento de Medicina, Universitat Autonoma de Barcelona, [Serial online] Septiembre 2004, Disponible en URL: http://www.tdx.cbuc.es/TESIS_UAB/AVAILABLE/TDX0728105-133440//emn 1 de1.pdf.

\section{SCHUMACHER K, STEWART B y ARCHBOLD} P, Conceptualización y medición de la ejecución de ser un buen cuidador familiar. En: Journal of Nursing Scholarship. New York. Vol 30, № 1 (1998) p. 6

12. VEGA Angarita, Olga Marina, Habilidad del cuidado de los cuidadores primarios de adultos discapacitados por enfermedad Cerebrovascular del Norte de Santander 2005, Trabajo de grado para obtener el titulo de Magíster en enfermería con énfasis al paciente Crónico, Universidad Nacional de Colombia-Universidad Francisco de Paula Santander.

13. GONZALEZ, Escobar Dianne Sofía, Habilidad del Cuidado De cuidadores familiares de personas en situación crónica de enfermedad por Diabetes Mellitus 2005, Trabajo de grado para obtener el titulo de Magíster en enfermería con énfasis al paciente 16
Crónico, Universidad Nacional de ColombiaUniversidad Francisco de Paula Santander.

14. RAMIREZ Ordóñez $M^{a}$ Mercedes, Habilidad de cuidado de los cuidadores principales en el paciente con cáncer gastrointestinal 2005, Trabajo de grado para obtener el titulo de Magíster en enfermería con énfasis al paciente Crónico, Universidad Nacional de Colombia-Universidad Francisco de Paula Santander.

15. CHÁVEZ Cañas, Wilmer; ESTÉVEZ López, Erica P.; RUEDA Barranco, Julieth J. "Grado de preparación para el cuidado en casa de cuidadores familiares de adultos en situación de enfermedad crónica discapacitante Neurovascular de la ESE Hospital Universitario Erasmo Meoz durante el período comprendido entre Marzo y Julio de 2005". Trabajo de grado para optar al título de enfermera(o), Programa de Enfermería, Facultad de Ciencias de la Salud, Universidad Francisco de Paula Santander, p.95

16. CELMA Vicente Matilde, Cuidados Informales en el Medio Hospitalario, En: Revista Rol de enfermería, Volumen 24 (7-8) Julio-Agosto 2001, España, P. 504

17. ANZOLA P Elías, GALINSKY David, MORALES M, Fernando, Op Cite p.366

18. BARRERA Ortiz, Lucy, BLANCO De Camargo Lidia, FIGUEROA Ingrid $P$, et al. Habilidad de Cuidadores familiares con enfermedad crónica. Mirada Internacional. En revista Aquichan. 2006, vol.6, no. 1 (6), p 22-33

19. BARRERA O, Lucy, PINTO A, Natividad, SANCHEZ $\mathrm{H}$, Beatriz. Habilidad de Cuidado de Cuidadores Familiares de personas con enfermedad Crónica: Comparación de Géneros. En Revista: Actualizaciones de Enfermería 2006,9(2):9 -13. Fundación Santa Fe de Bogotá. http://encolombia.com/medicina/ enfermeria/Enfermeria9206-Habilidad.htm

20. BARRERA Ortiz, Lucy, BLANCO De Camargo 


\section{COMPARACIÓN ENTRE LA HABILIDAD DE CUIDADO DE CUIDADORES DE PACIENTES CON ENFERMEDAD} CEREBROVASCULAR Y DIABETES MELLITUS EN SAN JOSÉ DE CÚCUTA, NORTE DE SANTANDER.

Lidia, FIGUEROA Ingrid P, et al. Op. Cite p. 28-29

21. GALVIS LOPEZ, Clara Rocio, PINZON ROCHA, Maria Luisa, ROMERO GONZALEZ, Esperanza. Comparación entre la Habilidad de Cuidado de Cuidadores de personas en situación de enfermedad crónica en Villavicencio. En: Revista Avances en Enfermería Volumen XXII No 1 (Enero a Junio) 2004. P. 16

22. NKONGHO, NGOZI O. Inventario para la habilidad de cuidado. En Strickland O. Waltz C. Measurement of Nursing Outcomes. Traducción Facultad de Enfermería. Universidad Nacional de Colombia. 1.999. P. 5

23. QUEMADA José Ignacio, Modelo de Atención a las personas con daño cerebral, Serie colección de documentos Técnicos, Instituto de Mayores de Servicios Sociales, [Serial online] Primera Edición [Citado Junio 2007] España, Disponible en URL: http://www.imsersomayores.csic.es/documentos/ documentos/mtas-atencion-01.pdf, p. 64

24. FITZGERALD MILLER, Judith. Recursos de Poder del Cliente. Philadelphia. Davis, 2000. P.3-16

25. MARCO Navarro Esther, Calidad de vida del cuidador del hemipléjico vascular, Op. Cite. P. 64

Fecha de recibido: Octubre 31 de 2006.

Fecha de aceptación: Noviembre 30 de 2007 